\title{
Validity and reliability of ICIQ-MLUTS
}

\begin{abstract}
Sound assessment of lower urinary tract symptoms (LUTS) leads to better treatment outcomes. Several tools have been developed to help physicians understand the most bothersome symptom that the patient is suffering from. Of these tools, self-reporting questionnaires have been validated for use for this specific indication.
\end{abstract}

Keywords: ICIQ, LUTS, assessment
Volume 4 Issue 2 - 2017

\author{
Haitham Abdelmoteleb \\ Department of Urology, Great Western Hospital, UK
}

Correspondence: Haitham Abdelmoteleb, Department of Urology, Great Western Hospital, Swindon, SN3 6BB, UK, Tel 447884827995, Email haitham.abdelmoteleb@yahoo.com

Received: February 24, 2017 | Published: March 3, 2017
Abbreviations: ICS, international continence society; LUTS, lower urinary tract symptoms; ICI, international consultation on incontinence; ICIQ, international consultation of incontinence questionnaire; QoL, quality of life

\section{Introduction}

The International Continence Society (ICS) has set up a standard to the terminology of the lower urinary tract symptoms (LUTS). LUTS have been divided to storage, voiding and post micturition LUTS. Voiding LUTS comprise symptoms of slow stream, intermittency, hesitancy, splitting of stream, straining to void and terminal dribble. On the other hand, post micturition symptoms are comprised of sensation of incomplete bladder emptying and/or a post micturition dribble. While storage LUTS include increased daytime frequency, urgency, and nocturia and urine incontinence. ${ }^{1}$ This subcategorization of symptoms allows for a better understanding of the underlying pathophysiology leading to the patients' bother. A thorough assessment of lower urinary tract symptoms (LUTS) through categorizing patients' symptoms leads to better management outcome. In modern urology practice, tools have been developed to assist urologist in tailoring the management decision. One of the tools used to categorize LUTS are symptom questionnaires. That provides a subjective feedback of LUTS and supplies the treating physician with a comprehensive overview of the patients' symptoms.

In addition to the tools needed to assess LUTS; medical history, physical examination, bladder diaries and special tests, the International Consultation on Incontinence (ICI) highlights the importance of the use of high-quality questionnaires in the assessment of LUTS. The ICI gives a Grade a recommendation for the use of the International Consultation of Incontinence Questionnaire (ICIQ). ${ }^{2}$ The ICIQ has developed a comprehensive approach in assessing all LUTS, this is by weighting each symptom against its degree of bother to the patient. It is a questionnaire that was developed by the patients for the patients. It is more detailed than other questionnaires in common use and helps to reach a sound clinical judgment due to the valuable information it supplies. The main advantage of the ICIQ is that it enables the specification of the LUTS this is the most bothersome to the patient. ${ }^{3}$ One of the ICIQ is the ICIQ-MLUTS long form for the evaluation of the male LUTS and assess the impact on the quality of life (QoL). This is a twenty-three item patient completed questionnaire covering different aspects of male LUTS and includes a QoL question for each individual symptom. The ICIQ-MLUTS has been translated into over sixteen different languages and dialects. ${ }^{4}$ The validity and reliability of the ICIQ-MLUTS has been tested and proven on several occasions. Mainly, when it has been translated and back translated into English, also, when it has been compared to other questionnaires assessing LUTS such as the IPSS. In addition to that, it is easy to understand and handy to complete.

\section{Conclusion}

This highlights the importance of the use of patient-completed questionnaire as it provides the clinician with an in-depth assessment of the patients' bothersome symptoms and allows for a tailored management of each individual. Thus, patient-completed questionnaire should be used in the initial assessment and follow-up of treatment of every male patient presenting with LUTS.

\section{Acknowledgement}

None.

\section{Conflicts of interest}

The author declares there is no conflict of interest.

\section{References}

1. Abrams P, Cardozo L, Fall M, et al. The standardisation of terminology in lower urinary tract function: report from the standardisation sub-committee of the International Continence Society. Urology. 2003;61(1):37-49.

2. Abrams P, Andersson KE, Birder L, et al. Fourth International Consultation on Incontinence Recommendations of the International Scientific Committee : Evaluation and Treatment of Urinary Incontinence , Pelvic Organ Prolapse, and Fecal Incontinence. Neurourol Urodyn. 2010;29(1):213-240.

3. Abrams P, Avery K, Gardener N. The International Consultation on Incontinence Modular Questionnaire: www.iciq.net. J Urol. 2016;175(3 Pt 1):1063-1066.

4. ICIQ | MLUTS Long Form. http://www.iciq.net/ICIQ.MLUTSlongform. html. 